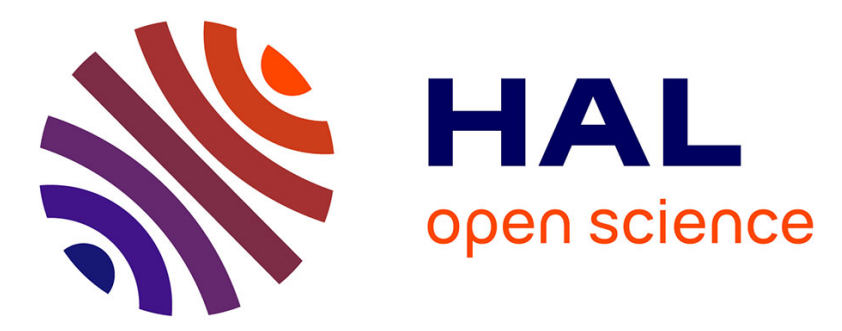

\title{
Experiments in the NH3-H2O system in the [0,1 GPa] pressure range - implications for the deep liquid layer of large icy satellites
}

\author{
O. Mousis, J. Pargamin, O. Grasset, C. Sotin
}

\section{- To cite this version:}

O. Mousis, J. Pargamin, O. Grasset, C. Sotin. Experiments in the NH3-H2O system in the $[0,1 \mathrm{GPa}]$ pressure range - implications for the deep liquid layer of large icy satellites. Geophysical Research Letters, 2002, 29 (24), pp.45-1 45-4. 10.1029/2002GL015812 . hal-00119108

\author{
HAL Id: hal-00119108 \\ https://hal.science/hal-00119108
}

Submitted on 26 Jan 2021

HAL is a multi-disciplinary open access archive for the deposit and dissemination of scientific research documents, whether they are published or not. The documents may come from teaching and research institutions in France or abroad, or from public or private research centers.
L'archive ouverte pluridisciplinaire HAL, est destinée au dépôt et à la diffusion de documents scientifiques de niveau recherche, publiés ou non, émanant des établissements d'enseignement et de recherche français ou étrangers, des laboratoires publics ou privés. 


\title{
Experiments in the $\mathrm{NH}_{3}-\mathrm{H}_{2} \mathrm{O}$ system in the [0, $\left.1 \mathrm{GPa}\right]$ pressure range - implications for the deep liquid layer of large icy satellites
}

\author{
Olivier Mousis, ${ }^{1}$ Judith Pargamin, Olivier Grasset, and Christophe Sotin \\ Laboratoire de Planétologie et Géodynamique, CNRS-UMR 6112, Nantes, France
}

Received 4 July 2002; revised 17 September 2002; accepted 17 September 2002; published 26 December 2002.

[1] We have experimentally studied the peritectic's melting temperature of a $25 \mathrm{wt} \%$ ammonia water solution in the $[0,1 \mathrm{GPa}]$ pressure range by using a high pressure low temperature membrane sapphire anvil cell with nitrogen cooled cryostat. Our experimental results are in agreement with those presented by Hogenboom et al. [1997] in the [0, $0.4 \mathrm{GPa}$ ] pressure domain where the peritectic's melting temperature follows a plateau at $180 \mathrm{~K}$. The present experimental work fills the lack of data between 0.4 and $0.7 \mathrm{GPa}$. It also suggests that the peritectic's melting temperature is much higher than that between 0 and $0.4 \mathrm{GPa}$ and does not vary very much with pressure at least up to 1 GPa. This implies the existence of two new high pressure phases in the ammonia-water system. The behaviour of the peritectic's melting curve above $0.5 \mathrm{GPa}$ might play an important role in the evolution of a deep liquid layer in the interiors of the largest icy satellites including Titan, Ganymede and Callisto. We show that the cooling of the deep liquid layer may have involved the crystallization of an ammonia bearing phase at higher temperature than previously expected. If the initial fraction of ammonia relative to ices is greater than 11 weight per cent, taking the partial ammonia depletion into account may lead to a final liquid layer thinner than previously inferred. INDEX TERMS: 6218 Planetology: Solar System Objects: Jovian satellites; 6280 Saturnian satellites; 6297 Instruments and techniques. Citation: Mousis, O., J. Pargamin, O. Grasset, and C. Sotin, Experiments in the $\mathrm{NH}_{3}-\mathrm{H}_{2} \mathrm{O}$ system in the $[0,1 \mathrm{GPa}]$ pressure range - implications for the deep liquid layer of large icy satellites, Geophys. Res. Lett., 29(24), 2192, doi:10.1029/ 2002GL015812, 2002.

\section{Introduction}

[2] Current thermochemical models developed for Jovian and Saturnian subnebulae [Prinn and Fegley, 1981; Mousis et al. 2002] predict that ammonia hydrate is stable in these environments. In the framework of these scenarios, ammonia, as well as other volatiles, was trapped as hydrate in grains and planetesimals before being accreted in icy satellites. Therefore, ammonia is presumed to be present in the interiors of icy satellites such as Titan, Ganymede or Callisto [Lunine and Stevenson, 1987; Grasset and Sotin,

\footnotetext{
${ }^{1}$ Also at Laboratoire de Physique des Isolants et d'Optronique, EA 3254, Université de Nantes, 2 rue de la Houssinière, F-44332 Nantes, France.
}

Copyright 2002 by the American Geophysical Union. 0094-8276/02/2002GL015812
1996]. The presence of ammonia in the interiors of large icy satellites probably implies the existence of a deep ocean because the solidus temperature is decreased by tens of degrees [Grasset et al., 2000].

[3] In order to constrain models of depth and thickness of such a deep ocean, a good knowledge of the high-pressure low-temperature phase diagram of the ammonia water mixture is required. The pressure field covered by the icy layers in large icy satellites such as Titan, Ganymede or Callisto being located below $1 \mathrm{GPa}$, some experimental and theoretical studies of the $\mathrm{NH}_{3}-\mathrm{H}_{2} \mathrm{O}$ phase diagram have been conducted in this domain [Johnson and Nicol, 1987, Lunine and Stevenson, 1987; Croft et al., 1988; Grasset et al., 1995; Hogenboom et al., 1997]. Nevertheless, an important lack of experimental data still exists in the pressure range [0.4, $0.7 \mathrm{GPa}$ [Hogenboom et al. 1997]. Consequently, the peritectic's melting curve of the $\mathrm{NH}_{3}$ $\mathrm{H}_{2} \mathrm{O}$ system remains weakly constrained in this pressure range.

[4] In this paper, new measurements of the peritectic's melting curve are presented in the $[0,1 \mathrm{GPa}]$ pressure range. The high-pressure and low-temperature apparatus (hereafter labelled HP-LT apparatus), as well as the experimental protocol are described in Section 2. Experimental results and comparisons with the previous data of Hogenboom et al. [1997] are presented in Section 3. Section 4 is devoted to the implications of our results on the characteristics of a deep liquid layer in large icy satellites.

\section{Experimental Protocol}

[5] Experiments are carried out in an optical sapphire anvil cell which allows in situ observations of phase transitions [Grasset, 2001]. Samples of $\mathrm{NH}_{3}-\mathrm{H}_{2} \mathrm{O}$ mixture are enclosed in a $200 \mu \mathrm{m}$ hole drilled in a pre-indented 60 $\mu \mathrm{m}$ thick stainless steel gasket $(\mathrm{Fe} / \mathrm{Cr} 18 / \mathrm{Ni10})$ which does not react with ammonia and provides an excellent resistance to deformation in the $[0,1 \mathrm{GPa}]$ pressure range. Ruby chips with $0.3 \% \mathrm{Cr}$ are incorporated in the sample and allow measurements of pressure through the analysis of their fluorescence [Piermarini et al., 1975; Grasset 2001]. The temperature range, lying between $77 \mathrm{~K}$ and $300 \mathrm{~K}$, is imposed by a cryostat cooled with liquid nitrogen. Temperature is controlled with thermocouples, which are placed close to the gasket. Modeling of temperature field within the cell shows that the standard deviation of temperature is from $\pm 0.7 \mathrm{~K}$ at $240 \mathrm{~K}$ to $\pm 1.2 \mathrm{~K}$ at 180 $\mathrm{K}$. Standard deviation for pressure is from $\pm 0.015 \mathrm{GPa}$ at $240 \mathrm{~K}$ to $\pm 0.035 \mathrm{GPa}$ at $180 \mathrm{~K}$. The present study investigates the phase changes of a 25 weight per cent 


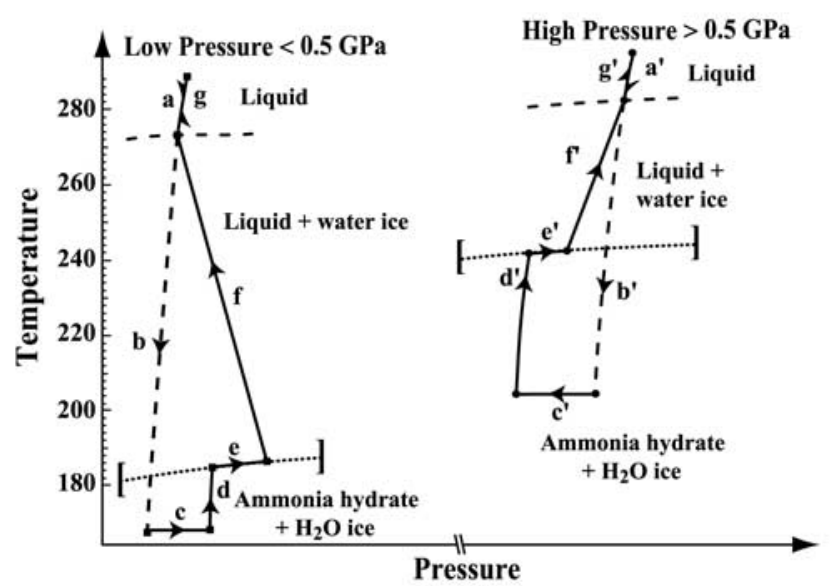

Figure 1. Experimental paths in the P-T diagram of the 25 wt $\%$ ammonia - water mixture for a fixed membrane pressure. The different experimental segments are labelled hereafter: $\left(a, a^{\prime}\right)$ cooling in the stable region of liquid $\left(b, b^{\prime}\right)$ cooling of the metastable liquid - $\left(c, c^{\prime}\right)$ crystallization with in-situ pressure variation $-\left(d, d^{\prime}\right)$ warming up of the solid - $\left(\mathrm{e}, \mathrm{e}^{\prime}\right)$ peritectic melting following the P-T equilibrium curve - $\left(f, f^{\prime}\right)$ warming up of a solid-liquid mixture $-\left(g, g^{\prime}\right)$ warming up of the liquid.

(hereafter labeled $w \mathrm{t} \%$ ) fraction of ammonia, just below the dihydrate composition $(\sim 32.1 \mathrm{wt} \%)$. Under its solid form, at room pressure, this mixture presents two coexistent crystalline phases: water ice and ammonia dihydrate [Hogenboom et al. 1997].

[6] After loading, the cell is tightly sealed in order to avoid ammonia volatilization and immediately settled in the cryostat. Before any experiment, a reference spectrum is acquired at ambient pressure for several incorporated ruby chips in order to calibrate future pressure measurements.

[7] Except during phase transitions, the sample enclosed in the gasket undergoes almost no volume change and follows segments of quasi-isochoric pathway in the $P-T$ space as long as the membrane pressure remains fixed. The quasi-isochoric pathway covered by the ammonia-water mixture during consecutive experiments is illustrated by Figure 1. First, the sample is cooled down to complete crystallization which occurs below the peritectic's melting curve temperature because of the important metastability of the system. At low pressure, ice I and ammonia dihydrate crystallize. Due to the low density of ice I, the pressure in the sample increases during crystallization (Figure 1 - segment c). At high pressure, the pressure in the sample decreases during crystallization because high-pressure ice polymorphs are denser than the liquid (Figure 1 - segment $c^{\prime}$ ). Sample is then slowly heated by steps of $2-3 \mathrm{~K}$. Between each step, the temperature is stabilized during several minutes in order to thermally equilibrate the sample. Once the first melting in the sample appears, fluorescence spectra are recorded for each ruby in order to compute the in situ pressure of the sample at the melting temperature $T_{m}$. The melting is interpreted as a peritectic melting following the reaction:

ammonia - ice polymorph $=$ ice + liquid.
[8] The sample remains at the peritectic equilibrium until the ammonia bearing phase has completely melted (Figure 1 - segments e and e'). Finally, ammonia-water liquid and ice in equilibrium get heated until the liquidus is reached (Figure 1 - segments $\mathrm{f}$ and $\mathrm{f}^{\prime}$ ).

[9] Spectroscopic characterizations were not possible during these experiments and no visible criteria have been found for distinguishing the different ammonia polymorphs. However, according to Hogenboom et al. [1997], ammonia dihydrate I and ammonia dihydrate II have been identified in the low-pressure range.

\section{Results}

[10] More than 50 runs have been carried out, including 24 in the $[0.4,0.7 \mathrm{GPa}]$ pressure range for which no data were available [Hogenboom et al., 1997]. Figure 2 illustrates the $25 \mathrm{wt} \%$ ammonia water peritectic transition in the $[0,1 \mathrm{GPa}]$ pressure range. The metastability of the ammonia-water melt causes a relative dispersion of our data, especially at high pressure. The formation of the first liquid is interpreted as the peritectic melting (half filled circles on Figure 2). These data suggest an abrupt change of the peritectic's melting temperature around $0.5 \mathrm{GPa}$. They also suggest that it depends weakly on pressure at low $P(0<P<$ $0.5 \mathrm{GPa})$ and high $P(0.5<P<1 \mathrm{GPa})$. The high pressure plateau presents a melting temperature around $232 \mathrm{~K} \pm 6 \mathrm{~K}$, which is approximately $50 \mathrm{~K}$ higher than that of the lower pressure plateau. According to the sensitivity of our equipment, the pressure corresponding to the temperature shift between these two levels lies in between 0.45 and $0.55 \mathrm{GPa}$.

[11] These results can be compared to those previously covered by Hogenboom et al. [1997] on Figure 2. These authors have reported the discovery of high pressure polymorphs of ammonia hydrates and reconciled previous data obtained by the UCLA and Lafayette groups. Our data, obtained in the pressure range $[0-0.4 \mathrm{GPa}]$, are in good agreement with those of the Lafayette team. This point indicates that the protocol followed in our experiment avoids the formation of the metastable eutectic water + ammonia monohydrate which may induce a significantly different melting curve. Thus, we believe that the system is composed, before melting, of the two stable phases: ice and ammonia dihydrate.

[12] In the high pressure domain, the peritectic melting curve obtained from our data is up to $40 \mathrm{~K}$ higher than that interpolated by Hogenboom et al. [1997] in the [0.4, 0.7 $\mathrm{GPa}$ ] pressure field, where no data were available. At 0.8 $\mathrm{GPa}$ and $1 \mathrm{GPa}$, our measurements are respectively about $20 \mathrm{~K}$ and $10 \mathrm{~K}$ higher than those of the UCLA group. These important discrepancies cannot be explained by the lack of precision of the different apparatus. The HP plateau can be due to either a peritectic composition change or the existence of a new phase. Hogenboom et al. [1997] have shown that the peritectic composition is enriched in ammonia when pressure increases above $0.2 \mathrm{GPa}$. This property may explain a transition to a low temperature plateau (eutectic system) but cannot account for the HP plateau observed in our experiments. Thus, we believe that the transition around $0.5 \mathrm{GPa}$ must be explained by the existence of two new phases (NP I and NP II on Figure 2). This is the only way to reconcile our data with those of the Lafayette group. 


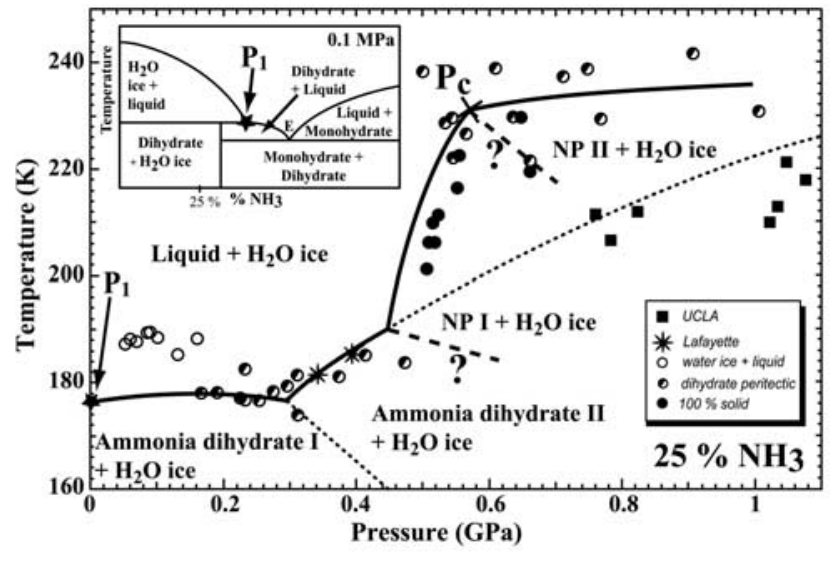

Figure 2. Peritectic's melting points measured in the $[0,1$ $\mathrm{GPa}$ ] pressure range of a $25 \mathrm{wt} \%$ ammonia hydrate sample. The solid bold curves correspond to the peritectic $P_{1}$ path in the $(P, T)$ field inferred from both our measurements and those of Lafayette group [Hogenboom et al., 1997]. In the low pressure range (up to $0.4 \mathrm{GPa}$ ), data from Lafayette group are labelled with black stars. The dotted line corresponds to the ammonia dihydrate I - ammonia dihydrate II transition described by Hogenboom et al. [1997]. In the high pressure range, the dotted curves and the associated symbols (black squares) represent measurements obtained by the UCLA group [Hogenboom et al., 1997]. NPI and NPII are the two ammonia hydrate high pressure phases discussed in Section 3. $P_{c}$ is the critical point localised at $[232 \mathrm{~K}, 0.5 \mathrm{GPa}]$. The frame on the left handside displays a sketch of the ammonia-water phase diagram at ambient pressure.

However these observations need to be confirmed by further experiments. The peritectic's melting curve between 0.45 and $0.55 \mathrm{GPa}$ exhibits a very strong value of $d T / d P$ which indicates that the NP I phase must be strongly different from the other hydrates which present slight melting temperature shifts with pressure. Unfortunately, this NP I phase has not been characterized in our experiments.

\section{Implications for Large Icy Satellites: The Case of Titan}

[13] In order to constrain the internal structure of large icy satellites such as Titan, the role of ammonia has to be taken into account. Titan, after homogenous accretion, underwent a core overturn which separated a silicate core surrounded by a shell of volatile rich liquid, including $\mathrm{NH}_{3}$ [Lunine and Stevenson, 1987; Grasset and Sotin, 1996]. After a rapid cooling, the surface temperature of Titan reached the crystallization point of ice at low pressure [Cassen et al. 1982]. Then, a ice I layer appeared at the surface of Titan and its thickness increased until it became sufficiently thick for convective instability to occur. At the base of the liquid layer, an icy layer should exist because of the intersection of the adiabatic temperature profile in the liquid layer with the liquidus curve of high pressure ices (Figure 3).

[14] One of the most critical parameter controlling the evolution of the liquid shell is its composition. The presence of ammonia decreases the freezing temperature and favors the existence of a liquid layer [Grasset and Sotin, 1996]. The present data are used to assess the thickness of the ocean. Once the thick liquid layer is trapped between the low and high pressure polymorphs of ice, its evolution is mainly driven by the balance between the energy produced in the silicate core due to the decay of the radiogenic elements and the heat which can be transferred by subsolidus convection through the outer ice I layer. If $\mathrm{NH}_{3}$ is present, the liquid layer cannot solidify because the $\mathrm{NH}_{3}$ compound decreases the solidification temperature of the liquid layer to values where the viscosity of ice is very large and impedes vigorous motions in the ice I lid [Grasset et al., 2000].

[15] The liquidus curves constraining the thickness of the deep liquid layer in Titan can be computed as follows. An initial ammonia-water composition is chosen. As the icy layers grow, all the ammonia remains in the liquid layer. Therefore, the ammonia composition is inversely proportional to the volume of the liquid layer:

$$
\frac{X}{X_{0}}=\frac{V_{0}}{V} \cdot \frac{\rho_{0}}{\rho} \approx \frac{V_{0}}{V},
$$

where $X, V, \rho$ correspond respectively to the ammonia composition ( $\mathrm{wt} \%$ ), volume and density of the liquid layer. Subscripts 0 correspond to the initial state. Assuming that density in the liquid layer remains constant during the solidification, we use the equation above in its simplest form. For a given value of $X_{0}$, the volume of remaining liquid is calculated at each $X$. Positions of both liquid-icy layers interfaces are then determinated by following an iterative procedure which consists to let converge $T, P$ values at each boundary towards those of the liquidus phase transition at $X$ in the ammonia-water phase diagram [Hogenboom et al. 1997]. This procedure is carried out for an ammonia composition ranging from $X_{0}$ to the eutectic composition in the remaining liquid layer. The resulting liquidus curves, which describe the $(P, T)$ curves at the solid/ liquid interface, take into account the evolution of pressure, temperature and composition in the liquid layer for the given initial composition $X_{0}$ through the crystallization process.

[16] The curves shown on Figures $3 \mathrm{a}$ and $3 \mathrm{~b}$ correspond to $X_{0}=5 \mathrm{wt} \%$ and $X_{0}=15 \mathrm{wt} \%$ respectively. In these figures, liquidus curves of the ammonia-water system in Titan are plotted with our peritectic curve superimposed. The temperature in the liquid layer is supposed to follow an adiabatic profile.

[17] With time, temperature in the liquid layer decreases as heat is evacuated through the ice I layer. Consequently, thicknesses of both ice I and high pressure ices layers increase to a value corresponding to the intersection of the thermal profile and the liquidus curve. As the temperature decreases, heat transfer through ice I becomes less and less efficient because viscosity increases [Grasset et al., 2000]. However, temperatures as low as $225 \mathrm{~K}$ can be obtained for plausible viscous characteristics of ice I as it is described in Deschamps and Sotin [2001]. In the $5 \mathrm{wt} \%$ case (Figure 3a), the liquidus curve is always right of the peritectic's melting curve. Only low and high pressure $\mathrm{H}_{2} \mathrm{O}$ ices exist as sketched on the right part of the figure. In the $15 \mathrm{wt} \%$ case (Figure 3b), as the temperature of the liquid layer decreases at values lower than that of the high pressure plateau of the peritectic's melting curve, high pressure ammonia hydrate and ice crystallize. When the base of the liquid layer reaches 

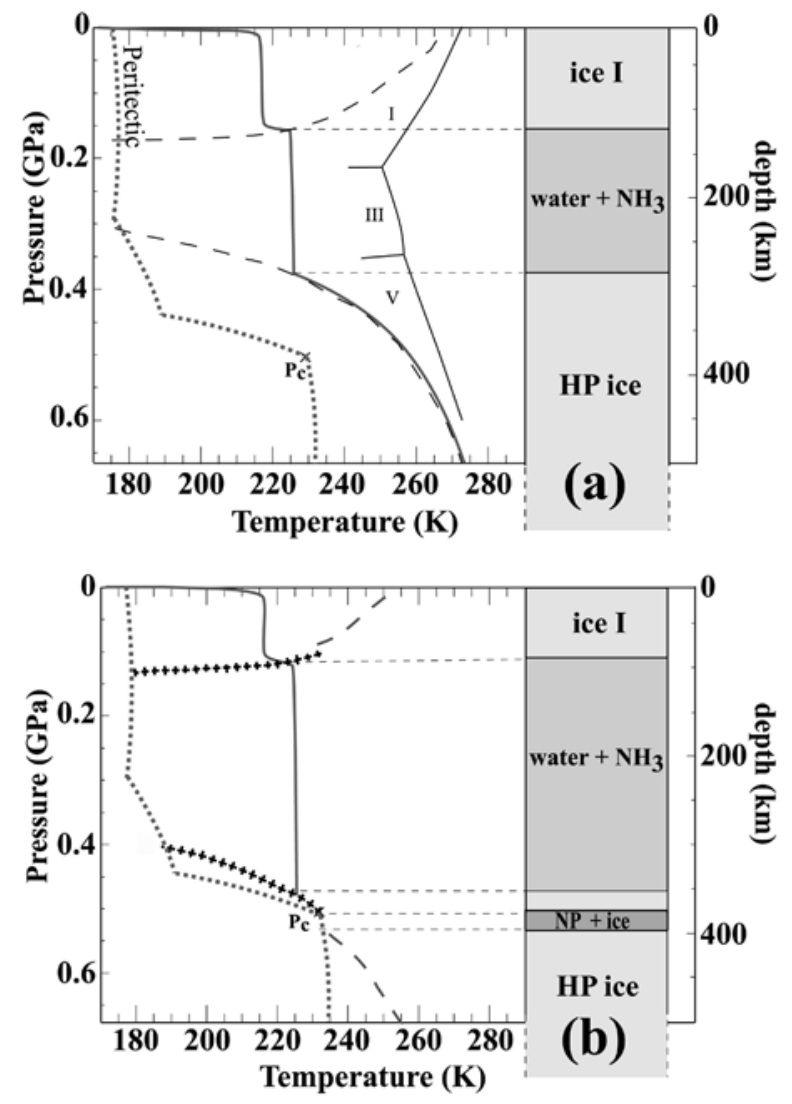

Figure 3. Implications of the experimental results on the evolution of the thickness of a deep liquid layer of Titan. The plain line describes the internal thermal profile, the temperature of the liquid is set at $225 \mathrm{~K}$. The dotted curve corresponds to the peritectic's melting curve plotted in Figure 2. (a) The dashed curves represent the liquidus curve for an initial composition of $5 \mathrm{wt} \%$ in ammonia. Thicknesses are computed for the represented thermal profile. Water ices phase diagram is also drawn for better comprehension. (b) The dashed curve is the liquidus curve for an initial composition of $15 \mathrm{wt} \%$ in ammonia; the crossed curve is the liquidus curve for an initial critical composition $(\sim 11 \mathrm{wt} \%)$.

the pressure of $0.5 \mathrm{GPa}$ ( point $P_{c}$ ), ammonia hydrate no longer crystallizes. The remaining liquid is depleted in ammonia, and crystallizes into ices, following a liquidus curve starting from the critical point $P_{c}$. This liquidus curve corresponds to a critical initial ammonia composition close to $11 \mathrm{wt} \%$. Any initial composition richer than this value will lead to the presence of a peritectic solid layer.

[18] If the initial fraction of ammonia relative to ices is greater than $11 \mathrm{wt} \%$, the scenario described above differs from previous models which predict that the liquid cools down without ammonia depletion, following the liquidus [Grasset and Sotin 1996]. Actually, taking the ammonia depletion into account leads to a final liquid layer thinner than previously inferred if its temperature decreases below $232 \mathrm{~K}$, the high pressure peritectic's melting temperature. For example, if the temperature of an initial $15 \mathrm{wt} \%$ ammonia rich liquid layer decreases down to $230 \mathrm{~K}$, its resulting thickness must be about 260 kilometers, rather than 335 kilometers. Moreover, at temperatures below 232 $\mathrm{K}$, any liquid layer with an initial composition richer than the critical composition (11 wt \%) will undergo an ammonia depletion leading to the same thickness and composition.

\section{Conclusions}

[19] We have shown that the peritectic's melting temperature of a $25 \mathrm{wt} \%$ ammonia-water mixture undergoes a temperature jump between 0.45 and $0.55 \mathrm{GPa}$ which probably implies the presence of two new high pressure phases. Our experimental data are from 30 to $40 \mathrm{~K}$ higher in the $[0.5,1 \mathrm{GPa}]$ pressure range than the peritectic's melting curve proposed by Hogenboom et al. [1997] based on previous data obtained at pressures larger than $0.8 \mathrm{GPa}$ [Cynn et al., 1989; Boone and Nicol, 1991]. The present experimental results suggest that the liquid layer of Titan is likely to be thinner than expected in previous works. Indeed, above a $11 \mathrm{wt} \%$ initial ammonia-water composition, an ammonia depletion occurs in the liquid layer due to the crystallization of a high pressure ammonia hydrate with the high pressure ices. The presence of such a deep liquid layer may be detected by gravity measurements made by the Cassini-Huygens mission [Castillo et al., 2000].

\section{References}

Cassen, P. M., S. J. Peale, and R. T. Reynolds, Structure and thermal evolution of the galilean satellites, In Satellites of Jupiter edited by D. Morrison, pp. 93-128, University of Arizona Press, Tucson, 1982.

Castillo, J., A. Mocquet, and C. Sotin, Détecter la présence d'un océan dans Europe à partir de mesures altimétriques et gravimétriques, C. R. Acad. Sci., 330(IIa), 659-666, 2000.

Croft, S. K., J. I. Lunine, and J. S. Kargel, Equations of state of ammoniawater liquid: Derivation and planetological applications, Icarus, 73, 279-293, 1988.

Deschamps, F., and C. Sotin, Thermal convection in the outer shell of large icy satellites, J. Geophys. Res., 106, 5107-5122, 2001.

Grasset, O., Calibration of the R ruby fluorescence lines in the pressure range $[0,1 \mathrm{GPa}]$ and the temperature range [250-300 K], High Press. Res., 21, 139-157, 2001.

Grasset, O., C. Sotin, and F. Deschamps, On the internal structure and dynamics of Titan, Planet. Space Science, 48, 617-636, 2000.

Grasset, O., and C. Sotin, The cooling rate of a liquid shell in Titan's interior, Icarus, 123, 101-112, 1996.

Grasset, O., C. Sotin, and S. Beauchesne, Etude par spectroscopie Raman in-situ du diagramme de phase de $\mathrm{NH}_{3}-\mathrm{H}_{2} \mathrm{O}$ dans le domaine $100 \mathrm{Mpa}-$ $1.5 \mathrm{GPa}$ : Application à la dynamique de Titan, C. R. Acad. Sci., 320(IIa), 249-256, 1995.

Hogenboom, D. L., J. S. Kargel, G. J. Consolmagno, T. C. Holden, and M. Buyyounouski, The ammonia-water system and the chemical differentiation of icy satellites, Icarus, 128, 171-180, 1997.

Johnson, M. L., and M. Nicol, The ammonia-water phase diagram and its implications for icy satellites, J. Geophys. Res., 92, 6339-6349, 1987.

Lunine, J. I., and D. J. Stevenson, Clathrate and ammonia hydrates at high pressure - Application to the origin of methane on Titan, Icarus, 70, 6177, 1987.

Mousis, O., D. Gautier, and D. Bockélée-Morvan, A turbulent model of Saturn's subnebula: Implications on the origin of the atmosphere of Titan, Icarus, 156, 162-175, 2002.

Piermarini, G. J., S. Block, J. D. Barnett, and R. A. Forman, Calibration of the pressure dependance of the R1 ruby florescence line to $195 \mathrm{kbar}$, J. Appl. Phys., 46(6), 2774-2780, 1975.

Prinn, R. G., and B. Fegley Jr., Kinetic inhibition of $\mathrm{CO}$ and $\mathrm{N}_{2}$ reduction in circumplanetary nebulae - Implications for satellite composition, Astrophys. J., 249, 308-317, 1981.

O. Mousis, J. Pargamin, O. Grasset, and C. Sotin, Laboratoire de Planétologie et Géodynamique, CNRS-UMR 6112, Université de Nantes, 2, rue de la Houssinière, F-44332 Nantes, France. (olivier.mousis@physique. univ-nantes.fr; Judith.Pargamin@chimie.univ-nantes.fr; Olivier.Grasset@ chimie.univ-nantes.fr; Christophe.Sotin@chimie.univ-nantes.fr) 